\title{
Tigecycline in the Treatment of Ventilator-Associated Pneumonia Due to Stenotrophomonas maltophilia: A Multicenter Retrospective Cohort Study
}

Lei Zha (D) - Dayan Zhang · Lingling Pan · Zhichu Ren •

Xiang Li · Yi Zou · Shirong Li · Shuangqi Luo · Gang Yang •

Boris Tefsen

Received: June 16, 2021 / Accepted: July 27, 2021 / Published online: August 10, 2021

(C) The Author(s) 2021

\section{ABSTRACT}

Introduction: Tigecycline is a potential alternative to trimethoprim-sulfamethoxazole in treating Stenotrophomonas maltophilia infections due to its potent in vitro antimicrobial activity. Clinical evidence regarding the use of tigecycline in the treatment of $S$. maltophilia

Supplementary Information The online version contains supplementary material available at https:// doi.org/10.1007/s40121-021-00516-5.

L. Zha

Emergency and Critical Care Unit, Conch Hospital of Anhui Medical University, Wuhu 241000, Anhui, China

\section{Zha $(\bowtie) \cdot$ B. Tefsen $(\bowtie)$}

Department of Biological Sciences, Xi'an JiaotongLiverpool University, No. 111, Ren'ai Road, Dushu Lake Higher Education Town, Suzhou Industrial

Park, Suzhou 215123, Jiangsu, China

e-mail: Lei.zha@liverpool.ac.uk

B. Tefsen

e-mail: boris.tefsen@ronininstitute.org

L. Zha

Institute of Infection and Global Health, University of Liverpool, Liverpool L69 7BE, UK

D. Zhang $\cdot$ Z. Ren $\cdot$ X. Li · Y. Zou

Postgraduate School, Wannan Medical College,

Wuhu 241000, Anhui, China infections is scarce. In this study, we assessed the efficacy of tigecycline treating ventilatorassociated pneumonia (VAP) due to $S$. maltophilia in comparison with fluoroquinolones.

Methods: This is a multicenter retrospective cohort study of patients admitted between January 2017 and December 2020 with the diagnosis of VAP caused by $S$. maltophilia receiving either tigecycline or fluoroquinolones as the definitive therapy $\geq 48 \mathrm{~h}$. Clinical outcomes including 28-day mortality, clinical cure and microbiological cure were analyzed.

L. Pan

Cardiology Department, The First Affiliated Hospital of Wannan Medical College, Wuhu 241000, Anhui, China

S. Li · G. Yang

Pulmonary and Critical Care Department, The Second People's Hospital of Wuhu, Wuhu 241000, Anhui, China

S. Luo Intensive Care Unit, The First People's Hospital of Wuhu, Wuhu 241000, Anhui, China

B. Tefsen Ronin Institute, Montclair, NJ 07043, USA 
Results: Of 82 patients with S. maltophilia VAP included, 46 received tigecycline, and 36 received fluoroquinolones; $70.7 \%$ of patients had polymicrobial pneumonia, and the appropriate empiric therapy was applied to only $14.6 \%$ of patients. The overall 28-day mortality was $39 \%$. Compared with patients receiving fluoroquinolones, tigecycline therapy resulted in worse clinical cure $(32.6 \%$ vs. $63.9 \%$, $p=0.009)$ and microbiological cure $(28.6 \%$ vs. $59.1 \%, p=0.045)$, while there was no statistical difference between 28 -day mortality $(47.8 \%$ vs. $27.8 \%, p=0.105)$ in the two groups. Similar results were also shown in the inverse probability of treatment weighted univariable regression model and multivariable regression model. Conclusions: The standard dose of tigecycline therapy was associated with a lower clinical and microbiological cure rate but not associated with an increased 28-day mortality in patients with $S$. maltophilia VAP compared with fluoroquinolones. Considering the unfavorable clinical outcomes, we therefore recommend against using the standard dose of tigecycline in treating $S$. maltophilia VAP unless new clinical evidence emerges.

Keywords: Fluoroquinolones; Levofloxacin; Moxifloxacin; Multicenter retrospective cohort study; Stenotrophomonas maltophilia; Tigecycline; Ventilator-associated pneumonia

\section{Key Summary Points}

Why carry out this study?

Stenotrophomonas maltophilia has emerged as an important hospital-acquired pathogen in critically ill patients, and therapeutic options for its infections are limited because of its extensive antimicrobial resistance.

Trimethoprim-sulfamethoxazole and fluoroquinolones are widely used in treating S. maltophilia infections.

However, because of the high rate of adverse events and the increasing rate of antimicrobial resistance, alternative antibiotics are urgently needed.
Tigecycline is a potential alternative for $S$. maltophilia infections due to its potent in vitro activity. However, studies assessing the effect of tigecycline in treating $S$. maltophilia infections are limited.

\section{What was learned from the study?}

Compared with fluoroquinolones, the standard dose of tigecycline therapy resulted in worse clinical outcomes, with a lower rate of clinical and microbiological cure but no statistical difference in 28-day mortality in patients with ventilatorassociated pneumonia caused by $S$. maltophilia.

We recommend against using the standard dose of tigecycline in the treatment of $S$. maltophilia ventilator-associated pneumonia unless new clinical evidence emerges.

\section{INTRODUCTION}

Stenotrophomonas maltophilia, previously Pseudomonas maltophilia or Xanthomonas maltophil$i a$, has emerged as an important hospitalacquired pathogen in critically ill patients, causing pneumonia, blood-stream infection and, less frequently, skin and soft tissue infection as well as urinary tract infection $[1,2]$. It has been reported as one of the top ten pathogens responsible for intensive care unit-acquired pneumonia in European countries, accounting for $0.4-8.7 \%$ of all hospital-acquired pneumonia (HAP) [3]. Although the incidence of HAP caused by $S$. maltophilia is relatively low, the corresponding mortality was around 50\% [4], and even reported as high as $77 \%$ in some populations [5].

Therapeutic options for S. maltophilia are often limited because of its extensive intrinsic or acquired resistance to antibiotics commonly used in nosocomial infections, including cephalosporins, $\beta$-lactam/ $\beta$-lactamase inhibitors, aminoglycosides and carbapenems $[6,7]$. Trimethoprim-sulfamethoxazole is therefore 
considered the first choice because of its potent in vitro activity against $90 \%$ of all clinical isolates [8]. However, the high rate of allergy, side effects and intravenous drug shortage limits its clinical use. Another popular alternative for the treatment of $S$. maltophilia infections in clinical practice is fluoroquinolones because of their good in vitro activity, convenient availability and relatively lower rate of side effects $[7,8]$. The clinical effectiveness of fluoroquinolones has been assessed in a meta-analysis that indicated fluoroquinolones are an effective alternative to trimethoprim-sulfamethoxazole in the treatment of bacteremia and pneumonia caused by $S$. maltophilia [9].

However, resistance to fluoroquinolones has seen an alarming trend in $S$. maltophilia $[10,11]$. Global surveillance demonstrated a decreased susceptibility of $S$. maltophilia to levofloxacin from $83.4 \%$ during $2003-2008$ to $77.3 \%$ in 2011 $[12,13]$, and an even lower susceptibility rate was reported in Chinese surveys [14, 15]. Therefore, other alternative antibiotics are urgently required.

Tigecycline is one of the new tetracyclines with broad-spectrum antibacterial activity and has been widely used in the treatment of gramnegative bacteria with multidrug resistance, like Acinetobacter baumannii, and Klebsiella pneumoniae [16, 17]. It also presents good activity against $S$. maltophilia. A study testing tigecycline against a worldwide collection of clinical $S$. maltophilia strains reported a susceptibility rate of 95.5\% [18], and the SENTRY antimicrobial surveillance program conducted in the USA, Europe and the Mediterranean region during 2009-2012 demonstrated a similar susceptibility rate (96\%) [13]. Moreover, tigecycline also displayed good susceptibility against $S$. maltophilia, which is resistant to levofloxacin and/ or trimethoprim-sulfamethoxazole [19].

Clinical studies assessing the effectiveness of tigecycline in the treatment of $S$. maltophilia infections are limited. Apart from one study with a small sample size comparing trimethoprim-sulfamethoxazole with tigecycline in patients with all kinds of nosocomial infection [20], only two case reports reported the potential role of tigecycline in the treatment of $S$. maltophilia infections [21, 22]. As tigecycline is frequently used in China, we conducted this study to evaluate its effectiveness in treating ventilator-associated pneumonia (VAP) caused by $S$. maltophilia.

\section{METHODS}

\section{Study design}

This is a multicenter, retrospective, observational cohort study conducted in three tertiary teaching hospitals in Wuhu, Anhui, China, The First People's Hospital of Wuhu, The Second People's Hospital of Wuhu and The First Affiliated Hospital of Wannan Medical College. The medical records of patients with the diagnosis of VAP were reviewed from January 2017 to December 2020. The study was approved by the ethics committee of Xi'an Jiaotong-Liverpool University (reference number 19-01-05) and the institutional review board in each participating hospital, and the informed consent was waived because of the nature of the retrospective study.

\section{S. maltophilia VAP}

VAP was diagnosed according to the 2016 clinical practice guidelines by the infectious diseases society of America and the American Thoracic Society [23]. Patients with a new or progressive lung infiltrate after $48 \mathrm{~h}$ of tracheal intubation and manifesting one of the following criteria were considered as VAP: temperature $>38^{\circ} \mathrm{C} \quad$ or $<36.5^{\circ} \mathrm{C}$; leukocyte count $>10 \times 10^{12} / 1$ or $<4 \times 10^{12} / 1$; purulent endotracheal aspirate. Pathogens responsible for the episode of VAP were determined with quantitative culture of samples collected within $48 \mathrm{~h}$ before or after the onset of VAP (endotracheal aspirate $\geq 10^{5} \mathrm{CFU} / \mathrm{ml}$ or bronchoalveolar lavage $\geq 10^{4} \mathrm{CFU} / \mathrm{ml}$ ) $[24,25]$. S. maltophilia VAP was diagnosed when $S$. maltophilia was recovered at the concertation reaching the threshold of the corresponding specimens, irrespective of monomicrobial or polymicrobial infection. The identification of microorganism and susceptibility test of antibiotics were performed with the Vitek 2 system (bioMérieux) 
and interpreted according to the Clinical and Laboratory Standards Institute criteria [26].

\section{Participants}

Patients (age $>18$ years) with the diagnosis of VAP caused by $S$. maltophilia receiving either fluoroquinolones (levofloxacin or moxifloxacin) or tigecycline as the definitive therapy for $>48 \mathrm{~h}$ were eligible. Levofloxacin was administrated as $500 \mathrm{mg}$ twice daily, moxifloxacin was administrated as $400 \mathrm{mg}$ once daily, and $50 \mathrm{mg}$ tigecycline was used twice per day following a 100-mg loading dose. Dosage adjustments according to renal function were acceptable. Patients meeting the following criteria during their VAP course were excluded: received both fluoroquinolones and tigecycline concomitantly or sequentially; concomitant pathogen was susceptible to neither of the antimicrobial agents in the definitive therapy regimens; use of inappropriate antibiotics as the definitive therapy for $S$. maltophilia $\geq 48 \mathrm{~h}$ (defined as antibiotics used for which $S$. maltophilia strains were not susceptible based on antimicrobial susceptibility testing results [27]). In cases where patients experienced more than one episode of $S$. maltophilia VAP, only the first episode was included.

\section{Outcomes and Definitions}

The primary endpoint of the study was to assess the rate of clinical cure. Secondary endpoints investigated were 28-day mortality and microbiological cure. Clinical cure was defined as complete resolution of all signs and symptoms of pneumonia at 14 days after the initial given dose of target antibiotics (fluoroquinolones or tigecycline) [28]. Whenever patients died or were discharged within 14 days after the inclusion, the clinical cure was assessed by the end of antibiotic therapy. The microbiological cure was defined as the absence of $S$. maltophilia in the culture of specimens collected within 2 days before or after the follow-up time point the 14th day after the initial given dose of target antibiotics $[25,29]$. Patients who died or were discharged within 14 days were excluded from the microbiological cure analysis.

\section{Data Extraction}

Data extracted from the medical records included age, gender, the reason for ICU admission, comorbidities, Charlson comorbidity index score [30], severity of disease at the time of $S$. maltophilia VAP onset (Acute Physiology and Chronic Health Evaluation II (APACHE II) [31], duration of mechanical ventilation before the onset of S. maltophilia VAP, concomitant isolated bacteria, antibiotics, duration of antibiotic therapy targeting $S$. maltophilia, microbiological results, clinical cure and 28-day mortality. For patients discharged from hospitals earlier than 28 days after the onset of $S$. maltophilia VAP, information on 28-day mortality was obtained from their 1-month follow-up records.

\section{Statistical Analysis}

Continuous variables were summarized as median and interquartile range. Categorical variables were described as counts and percentages. The differences between patients receiving tigecycline or fluoroquinolones were analyzed with Fisher's exact test for categorical variables and Mann-Whitney $U$ test for continuous variables.

To analyze the clinical outcomes between tigecycline and fluoroquinolones in the treatment of VAP caused by $S$. maltophilia, an inverse probability of treatment-weighted (IPTW) univariable logistic regression model was performed [32]. The propensity score was estimated by using a nonparsimonious multivariable logistic regression model, with receiving tigecycline as the dependent variable and the baseline characteristics in the two groups with a standardized mean difference $>0.2$ as covariates [33]. The included covariates were severity of acute and chronic diseases (APACHE II score and Charlson comorbidity index score), comorbidities (malignancy, chronic liver failure, chronic heart failure, coagulation disorder), polymicrobial infection, co-isolated with $A$. baumannii, combination therapy with 
carbapenems and duration of antibiotic therapy targeting $S$. maltophilia.

Moreover, a stepwise forward multivariable regression model was run as a sensitivity analysis [34], by adjusting for variables determined a priori that were reported as the risk factors of mortality in $S$. maltophilia pneumonia and variables with $p \leq 0.2$ : age, gender, chronic kidney disease, coagulation disorder, malignancy, polymicrobial infection, definitive antibiotic therapy, combination therapy with carbapenems, APACHE II score and Charlson comorbidity index score. Subgroup analysis was also performed by excluding patients co-infected with Pseudomonas aeruginosa as this pathogen is naturally resistant to tigecycline [35]. Moreover, to remove the impact of appropriate initial antibiotic therapy on the assessment of effectiveness of antibiotic therapy, a subgroup analysis by excluding patients receiving appropriate empiric antibiotic therapy was also conducted.

Odds ratio and $95 \%$ confidence interval were reported. Two-tailed $p<0.05$ was considered statistically significant. All the statistical analyses were performed with $\mathrm{R}$ software version 3.6.2 (R Foundation for Statistical Computing).

\section{RESULTS}

\section{Patient's Characteristics}

Of the total of 142 patients with VAP caused by S. maltophilia meeting the inclusion criteria, 60 were excluded according to the exclusion criteria. The remaining 82 patients were included in the final analysis, among which 46 patients were treated with tigecycline, while the other 36 patients received fluoroquinolones levofloxacin or moxifloxacin (Fig. 1).

Patients with VAP caused by S. maltophilia were relatively old, with the median age of 76 (IQR 64.25-85) years, and had both serious acute and chronic diseases, with an APACHE II score of 21 (IQR 16.25-24) and a Charlson index comorbidity score of 5 (IQR 4-6). Most patients had at least one comorbidity, and hypertension was the most frequently reported comorbidity $(45 / 82, \quad 54.9 \%)$. A chronic underlying respiratory disease was present in 11 patients (13.4\%). Reasons for patients to be admitted to ICU were respiratory failure, stroke, sepsis, brain trauma, scheduled surgery and trauma.

VAP caused by $S$. maltophilia occurred late, with the median duration from intubation to VAP onset of 15 (IQR 9-33.75) days. A majority of patients $(58 / 82,70.7 \%)$ with $S$. maltophilia VAP were polymicrobial, with A. baumannii (26/ $58,44.8 \%)$ as the most commonly co-isolated bacterium, followed by Enterobacteriaceae (22/ 58, 37.9\%) and P. aeruginosa (10/58, 17.2\%). All patients with $S$. maltophilia VAP had received combination therapy at the onset of VAP by combining with either carbapenems (60/82, $73.2 \%)$ or $\beta$-lactam/ $\beta$-lactamase inhibitors $(22 /$ $82,26.8 \%)$. The duration of antibiotic therapy targeting S. maltophilia VAP was 9 (IQR 5.25-13) days. In terms of appropriate initial antibiotic therapy, only $14.6 \%(12 / 82)$ of the patients received effective antimicrobial therapy, and they were all in the fluoroquinolone therapy group.

Compared with patients receiving fluoroquinolones, patients receiving tigecycline were more likely to have a higher APACHE II score, Charlson comorbidity index score and longer time between the start of mechanical ventilation and the diagnosis of $S$. maltophilia VAP, albeit not statistically significant. Moreover, there were more patients receiving carbapenems in the tigecycline group, while more $\beta$-lactam $/ \beta$ lactamase inhibitors were used in patients receiving fluoroquinolones $(40 / 46,87 \%$ vs. 20/36, 55.6\%, $p=0.003$ ) (Table 1).

\section{Microbiological Profile}

Results of antimicrobial susceptibility testing were included for $S$. maltophilia isolates from 129 patients. The formal laboratory report of antimicrobial susceptibility testing of the remaining 13 patients cannot be found in the database, but part of the information (microorganism, quantitative culture result, whether susceptible to the prescribed antibiotics) was extracted from chart notes in their medical records. Therefore, the lack of formal testing reports in these patients did not influence the 


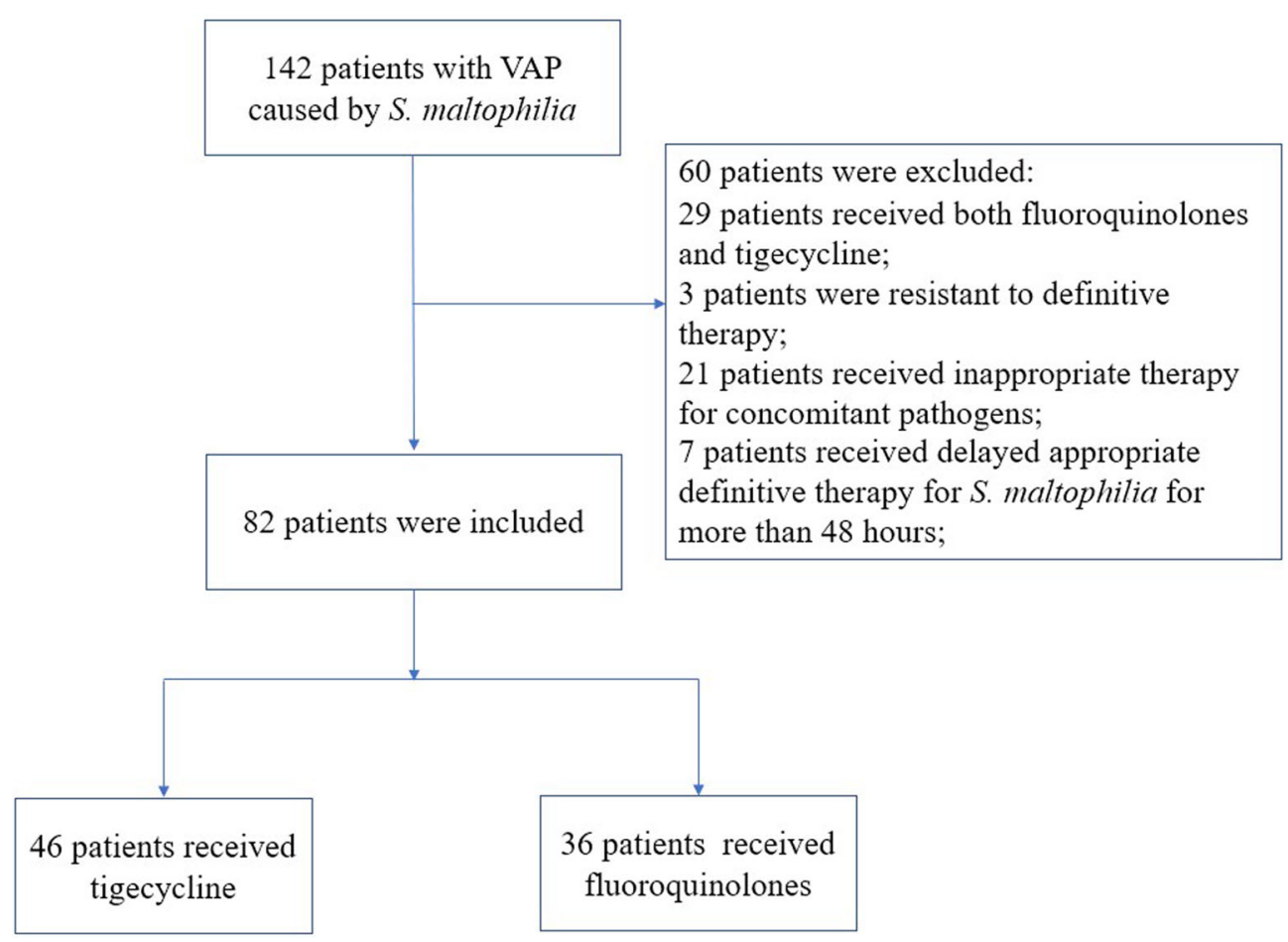

Fig. 1 Flowchart of study inclusion process

inclusion process and the final analysis. Tigecycline $(98.4 \%, 127 / 129)$ presented the highest susceptibility rate against $S$. maltophilia, followed by trimethoprim-sulfamethoxazole $(82.9 \%, 107 / 129)$ and levofloxacin (80.6\%, $104 / 129)$, while susceptibility rate was lower in ticarcillin-clavulanate and ceftazidime, $75.2 \%$ $(97 / 129)$ and $61.2 \%$ (79/129), respectively. No information regarding the susceptibility of $S$. maltophilia to carbapenems and $\beta$-lactam/ $\beta$-lactamase inhibitors was reported.

\section{Clinical Outcomes}

Compared with patients receiving fluoroquinolones, patients receiving tigecycline resulted in a lower clinical cure $(15 / 46,32.6 \%$ vs. $23 / 36,63.9 \%, p=0.009)$ and microbiological cure $(10 / 35,28.6 \%$ vs. $13 / 22,59.1 \%$, $p=0.045)$. There was no statistical difference in 28-day mortality between patients receiving tigecycline and fluoroquinolones (22/46, 47.8\% vs. $10 / 36,27.8 \%, p=0.105)$, although the trend is in favor of fluoroquinolones therapy (Table 2). In the IPTW univariable regression model, tigecycline therapy was associated with a reduced clinical cure $(0.75,95 \%$ CI $0.61-0.93$, $p=0.008)$, while it was not associated with an increased 28-day mortality $(1.14,95 \%$ CI 0.93-1.39, $p=0.195)$. Similar results were also shown in the multivariable logistic regression model that was run as the sensitivity analysis (Table 3 and Supplementary material, Table S1). Subgroup analysis by excluding patients co-infected with $P$. aeruginosa did not change the trend of any of these results. When patients receiving appropriate empiric antibiotic therapy were excluded, tigecycline therapy still resulted in a lower clinical cure $(15 / 46,32.6 \%$ vs. $15 / 24$, $62.5 \%, p=0.032$ ) than in patients receiving fluoroquinolones (Table 2).

\section{DISCUSSION}

Tigecycline is a promising alternative to trimethoprim-sulfamethoxazole in the treatment of $S$. maltophilia infections due to its potent antimicrobial activity [12]. Although resistance to tigecycline has been reported as an 
Table 1 Characteristics of patients with ventilator-associated pneumonia due to $S$. maltophilia receiving tigecycline or fluoroquinolones

\begin{tabular}{|c|c|c|c|c|}
\hline Variables & $\begin{array}{l}\text { All } \\
n=82\end{array}$ & $\begin{array}{l}\text { Tigecycline } \\
n=46\end{array}$ & $\begin{array}{l}\text { Fluoroquinolones } \\
n=36\end{array}$ & $p$ \\
\hline Age (year), median (IQR) & $\begin{array}{c}76[64.25, \\
85]\end{array}$ & $76[65,85]$ & $75[62.5,84.75]$ & 0.650 \\
\hline Male gender $n(\%)$ & $65(79.3)$ & $38(82.6)$ & $27(75)$ & 0.569 \\
\hline Reasons for ICU admission $n$ (\%) & & & & 0.134 \\
\hline Respiratory failure & $42(52.1)$ & $25(54.3)$ & $17(47.2)$ & \\
\hline Stroke & $17(20.7)$ & $8(17.4)$ & $9(25)$ & \\
\hline Sepsis & $8(9.8)$ & $6(13)$ & $2(5.6)$ & \\
\hline Brain trauma & $7(8.5)$ & $2(4.3)$ & $5(13.9)$ & \\
\hline Scheduled surgery & $6(7.3)$ & $5(10.9)$ & $1(2.8)$ & \\
\hline Trauma & $2(2.4)$ & $0(0)$ & $2(5.6)$ & \\
\hline \multicolumn{5}{|l|}{ Comorbidities $n(\%)$} \\
\hline Hypertension & $45(54.9)$ & $25(54.3)$ & $20(55.6)$ & 1.000 \\
\hline Coronary heart disease & $31(37.8)$ & $18(39.1)$ & $13(36.1)$ & 0.960 \\
\hline Chronic heart failure & $17(20.7)$ & $12(26.1)$ & $5(13.9)$ & 0.281 \\
\hline Chronic respiratory disease & $11(13.4)$ & $6(13)$ & $5(13.9)$ & 1.000 \\
\hline Chronic kidney disease & $7(8.5)$ & $5(10.9)$ & $2(5.6)$ & 0.648 \\
\hline Chronic liver disease & $10(12.2)$ & $7(15.2)$ & $3(8.3)$ & 0.545 \\
\hline Malignancy & $10(12.2)$ & $8(17.4)$ & $2(5.6)$ & 0.199 \\
\hline Diabetes mellitus & $15(18.3)$ & $8(17.4)$ & $7(19.4)$ & 1.000 \\
\hline Coagulation disorder & $10(12.2)$ & $8(17.4)$ & $2(5.6)$ & 0.199 \\
\hline Charlson comorbidity score, median (IQR) & $5[4,6]$ & $5[4,6]$ & $4[4,6]$ & 0.123 \\
\hline APACHE II score, median (IQR) & $\begin{array}{c}21[16.25, \\
24]\end{array}$ & $21.5[17,24.75]$ & $19.5[15.75,23]$ & 0.195 \\
\hline $\begin{array}{l}\text { Time from mechanical ventilation to } S \text {. maltophilia VAP (day), median } \\
\text { (IQR) }\end{array}$ & $15[9,33.75]$ & $\begin{array}{l}18.5[9.75 \\
34.75]\end{array}$ & $14.5[7,26.25]$ & 0.328 \\
\hline Polymicrobial infection $n$ (\%) & $58(70.7)$ & $35(76.1)$ & $23(63.9)$ & 0.387 \\
\hline A. baumannii & $26(44.8)$ & $18(51.4)$ & $8(34.8)$ & \\
\hline$P$. aeruginosa & $10(17.2)$ & $6(17.1)$ & $4(17.4)$ & \\
\hline Enterobacteriaceae & $22(37.9)$ & $11(31.4)$ & $11(47.8)$ & \\
\hline Concomitant antibiotics $n(\%)$ & & & & 0.003 \\
\hline Carbapenems & $60(73.2)$ & $40(87)$ & $20(55.6)$ & \\
\hline$\beta$-Lactam $/ \beta$-lactamase inhibitors & $22(26.8)$ & $6(13)$ & $16(44.4)$ & \\
\hline Duration antibiotic therapy targeting $S$. maltophilia & $9[5.25,13]$ & $9[5,13]$ & $9[7,13.25]$ & 0.533 \\
\hline Appropriate initial antibiotic therapy $n(\%)$ & $12(14.6)$ & $0(0)$ & $12(33.3)$ & $<0.001$ \\
\hline
\end{tabular}

increasing trend, the susceptibility rate in global surveillance reports is still high $(90.6 \%$ inhibited at $\leq 2 \mathrm{mg} / \mathrm{l})[36,37]$. Therefore, it was recommended as one of the antibacterial choices by the Chinese expert consensus statement to treat S. maltophilia infections since 2013 [38], especially for those critically ill patients or those concomitantly infected with other gram- 
Table 2 Clinical outcomes of patients with ventilator-associated pneumonia due to S. maltophilia receiving tigecycline or fluoroquinolones

\begin{tabular}{lcccc}
\hline Outcomes & All & Tigecycline & Fluoroquinolones & $p$ \\
\hline Overall analysis & & & \\
Clinical cure $(n=82)$ & $38(46.3 \%)$ & $15(32.6 \%)$ & $23(63.9 \%)$ & 0.009 \\
Microbiological cure $(n=57)$ & $23(40.4 \%)$ & $10(28.6 \%)$ & $13(59.1 \%)$ & 0.045 \\
28-Day mortality $(n=82)$ & $32(39 \%)$ & $22(47.8 \%)$ & $10(27.8 \%)$ & 0.105 \\
Subgroup analysis by excluding $P$. aeruginosa co-infection & & & 0.019 \\
Clinical cure $(n=72)$ & $35(48.6 \%)$ & $14(35 \%)$ & $21(65.6 \%)$ & 0.065 \\
28-Day mortality $(n=72)$ & $30(41.7 \%)$ & $21(52.5 \%)$ & $9(28.1 \%)$ & 0.032 \\
Subgroup analysis by excluding appropriate initial antibiotic therapy & & $15(62.5 \%)$ & 0.052 \\
Clinical cure $(n=70)$ & $30(42.9 \%)$ & $15(32.6 \%)$ & $5(20.8 \%)$ & \\
28-Day mortality $(n=70)$ & $27(38.6 \%)$ & $22(47.8 \%)$ & \\
\hline
\end{tabular}

negative bacteria, such as $A$. baumannii and $K$. pneumoniae, as these pathogens are usually presenting a high rate of resistance to other commonly used antibiotics in VAP [23]. In this study, patients with a higher APACHE II score and Charlson comorbidity index score were more likely to receive tigecycline and the combination therapy with carbapenems, indicating that clinicians are prone to prescribe tigecycline and combination therapy with other high-level antibiotics to those patients with greater severity of underlying diseases [39, 40]. Besides, coisolated with multidrug resistant or carbapenem-resistant bacteria was another factor that led doctors to use tigecycline in our study, as there were few choices for such infection.

In the present study, a lower rate of clinical cure and microbiological cure was reported in patients receiving tigecycline compared with patients receiving fluoroquinolones in the treatment of VAP caused by $S$. maltophilia. A reasonable explanation for the lower success rate of tigecycline in the treatment might be that the dose of tigecycline used in the study was not optimal, as a pharmacokinetic and pharmacodynamic (PK/PD) study has demonstrated that the standard dose of tigecycline (50 mg twice per day following a $100 \mathrm{mg}$ loading dose) resulted in a suboptimal concentration of tigecycline in the lung [41]. Moreover, clinical evidence also opposed the use of standard dose of tigecycline in treatment of severe infections caused by other pathogens than S. maltophilia, as it was associated with a poorer clinical outcome compared with the high-dose regimen or other comparators [16]. A study performed on 476 clinical isolates of $S$. maltophilia from a global collection revealed that $1 \mathrm{mg} / \mathrm{l}$ tigecycline was required to inhibit the growth of $50 \%$ of those isolates, and $2 \mathrm{mg} /$ $\mathrm{ml}$, to inhibit the growth of $90 \%$ of those isolates [36]. Combining this finding with a recent pharmacokinetics study of high-dose tigecycline (100 mg twice per day following a $200 \mathrm{mg}$ loading dose) in critically ill patients with severe infections caused by different organisms that indicated that only $40.6 \%$ and $28.1 \%$ of patients reached a concentration of tigecycline $\geq 1 \mathrm{mg} / \mathrm{l}$ and $2 \mathrm{mg} / \mathrm{l}$, respectively [42], it is improbable to expect a superior clinical outcome for high-dose tigecycline therapy. Therefore, whether high-dose tigecycline could result in better clinical outcomes than standard dose or other alternatives in the treatment of $S$. maltophilia pneumonia needs further scrutiny. On the other hand, it should be noted that the levofloxacin dose used in the present study (500 mg twice daily) was higher than 
Table 3 Analysis of clinical cure and 28-day mortality in patients with ventilator-associated pneumonia due to S. maltophilia receiving tigecycline or fluoroquinolones

\begin{tabular}{lll}
\hline Analysis (tigecycline vs. fluoroquinolones) & Odds ratio $\mathbf{9 5 \%} \mathbf{C I})$ & $\boldsymbol{p}$ \\
\hline Clinical cure & & 0.006 \\
Crude unadjusted analysis & $0.27(0.10-0.67)$ & 0.026 \\
Multivariable logistic regression & $0.32(0.12-0.86)$ & 0.008 \\
Inverse probability of treatment weighted model & $0.75(0.61-0.93)$ & 0.070 \\
28-Day mortality & & 0.355 \\
Crude unadjusted analysis & $2.38(0.96-6.23)$ & 0.195 \\
Multivariable logistic regression & $1.64(0.58-4.77)$ & $1.14(0.93-1.39)$ \\
Inverse probability of treatment weighted model &
\end{tabular}

recommended in clinical guidelines $(750 \mathrm{mg}$ once daily) [23], although the former has also been used in European countries in this way [29]. Studies in patients with severe lower respiratory tract infections have indicated that $500 \mathrm{mg}$ twice daily of levofloxacin was comparable to $400 \mathrm{mg}$ once daily moxifloxacin in PK/ PD profile, and both were superior to levofloxacin $500 \mathrm{mg}$ and $750 \mathrm{mg}$ once daily [43]. However, whether this superiority in PK/PD contributed to the better clinical outcomes in patients receiving fluoroquinolone is still uncertain, as there were no patients using levofloxacin $750 \mathrm{mg}$ once daily in the present study.

As it is well known that tigecycline is largely used as the salvage therapy for pathogens that are difficult to treat, it is rarely prescribed as an empirical therapy for patients with suspected VAP $[17,44]$. By contrast, fluoroquinolones are commonly recommended as empiric therapy for VAP [23]. Combined with the nature of extensive drug resistance of $S$. maltophilia against most of the commonly used antibiotics in the empiric therapy of VAP [7], a certain proportion of patients treated with tigecycline would have experienced the delayed appropriate initial antibiotic therapy compared with patients receiving fluoroquinolones. In the present study, patients in the tigecycline group all received tigecycline after the result of antimicrobial susceptibility testing was known, and only $14.6 \%$ of patients received appropriate initial antibiotic therapy, all in the fluoroquinolones group. Therefore, it is reasonable to speculate the lower clinical cure in tigecycline therapy might be due to the delay in adequate antibiotic therapy, as this has been well established in other multidrug-resistant bacterial infections [45-47]. However, when patients receiving appropriate empiric antibiotic therapy were excluded, the results did not change as well, indicating that inappropriate empiric antibiotic therapy cannot fully explain the lack of efficacy in the tigecycline group. Apart from the delayed use of appropriate antibiotics, another factor that might diminish the effectiveness of tigecycline is the relatively short course of antibiotic therapy, as it is a bacteriostatic agent [35]. Although guidelines now all recommend using a short course of antibiotic therapy for VAP, the duration for non-fermenting gram-negative bacteria seems insufficient $[23,48]$. A randomized trial compared a 7-day antibiotic therapy with a 10-day course indicating that patients with VAP caused by $P$. aeruginosa had a significantly higher 28-day mortality in the 7-day course [49]. Other studies also suggested that a longer course of antibiotic therapy is required to successfully treat VAP caused by multidrug-resistant gram-negative bacteria [50, 51]. Therefore, a longer course of tigecycline therapy for $S$. maltophilia VAP determined by integrating biomarkers and 
clinical assessment might be beneficial but still requires more evidence.

When interpreting the therapeutic effect of tigecycline in the treatment of $S$. maltophilia VAP, an important factor that needs to be considered is polymicrobial infection, as this reportedly accounted for $54.4-73.3 \%$ of $S$. maltophilia pneumonia in several studies $[4,39,52,53]$. In the present study, a similar result was also demonstrated, as $70.7 \%$ of patients were documented with polymicrobial infection. The pathogens co-isolated with $S$. maltophilia were consistent with these studies, with $A$. baumannii, Enterobacteriaceae and $P$. aeruginosa as the most frequently isolated. Studies assessing the efficacy of tigecycline in the treatment of other infections have shown that tigecycline was associated with poorer clinical outcomes compared with other antibiotics [54-56]. Importantly, $P$. aeruginosa is naturally resistant against tigecycline [35]. Therefore, it is better not to recommend the use of tigecycline in polymicrobial S. maltophilia $\mathrm{VAP}$, as the role of co-isolated pathogens in clinical course is still unclear, despite the studies that indicated no association between mortality and polymicrobial infection [4, 39, 52]. Paradoxically, the indication of using tigecycline to treat $S$. maltophilia VAP in the present study is usually the co-isolation of multidrug-resistant or carbapenem-resistant bacteria. Before 2019, the management strategies for these pathogens in the participating centers were restricted to tigecycline, double carbapenems and prolonged infusion of carbapenem or high-dose sulbactam, as polymyxins, minocycline, fosfomycin and other new antibiotics were not available at that time. Although we have implemented the IPTW model in the statistical analysis, trying to eliminate the indication bias, however, with the small sample size and nature of the retrospective study, we cannot incorporate all factors in the final analysis, especially the various resistance profiles of the co-isolated bacteria, which might ultimately influence the interpretation of the results.

It is interesting to note that even with a lower rate of clinical and microbiological cure in patients receiving tigecycline, the 28-day mortality was not statistically different from that of patients receiving fluoroquinolones, although the absolute risk difference was as high as $20 \%$. One possible explanation for this observation could be that the severity of underlying disease might contribute more to death than $S$. maltophilia VAP itself $[57,58]$, as patients developing $S$. maltophilia VAP shared common features with patients at high risk of death, like prolonged in-hospital stay and mechanical ventilation and a higher severity score of underlying disease $[5,52,59-61]$. Consistent results were also reported in other studies, indicating that the mortality of $S$. maltophilia pneumonia was independently associated with the severity of diseases (SOFA score) rather than factors related to antibiotic therapy, like a specific antimicrobial agent, appropriate initial therapy or whether being part of combination therapy [4, 39, 52, 53]. Moreover, the small sample size should also be considered when interpreting the no statistical difference in 28-day mortality, as the small sample in the present study might not be sufficiently powered to detect a difference between the groups and turn out to be falsely negative, leading to a type II error [62].

There are several limitations in this study. First, we aimed to compare the effectiveness between tigecycline and fluoroquinolones in the treatment of VAP infected with $S$. maltophilia, but were unable to remove the impact of concomitant antibiotics on clinical outcomes, especially the $\beta$-lactam/ $\beta$-lactamase inhibitors as they were reported to have around $50 \%$ susceptibility rate against S. maltophilia isolates in China [15]. Therefore, it is difficult to link clinical outcomes to one specific antibiotic. Second, $70.7 \%$ of patients included in this study had polymicrobial infection. Although patients concomitantly infected with a pathogen that was not susceptible to either antibiotic in the combination regimen were excluded, the various virulences and resistances of pathogens on the individual level still complicate the interpretation of the effectiveness of specific antibiotics in S. maltophilia VAP. Moreover, with the meager rate of blood culture implemented in the study cohort and different methods applied for collecting respiratory secretions, we failed to incorporate bacteremia and bacterial load in the 
final analysis. Third, because there were no patients receiving tigecycline as the empirical therapy for $S$. maltophilia VAP, we could not analyze the effectiveness of appropriate empiric therapy with tigecycline in these patients. Finally, although we tried to address the confounding by the IPTW model, and adjusted for variables through multivariable logistic regression, the small sample size prevents us from adjusting all variables, especially those unmeasured confounding factors that exist because of the nature of the multicenter observational retrospective study, like the heterogeneity in clinical practice, medical human resources and experience of medical staff among these centers, which diminish the power of statistics. Despite these limitations, this is the only study specifically focused on the assessment of clinical effectiveness of tigecycline in the treatment of VAP due to $S$. maltophilia. The results could be used as clinical evidence to support the choice of antibiotic therapy in S. maltophilia VAP.

\section{CONCLUSIONS}

Therapy with the standard dose of tigecycline in patients with VAP caused by $S$. maltophilia resulted in a significantly lower clinical and microbiological cure rate compared with treatment with fluoroquinolones. Considering the unfavorable clinical outcomes, we therefore recommend against using the standard dose of tigecycline in treating $S$. maltophilia VAP unless new clinical evidence emerges.

\section{ACKNOWLEDGEMENTS}

Funding. The study, including the Journal's Rapid Service Fees, was funded by Conch Hospital of Anhui Medical University (Wuhu, Anhui, China) as part of the research project on multidrug resistant bacteria (F20190301).

Authorship. All named authors meet the International Committee of Medical Journal Editors (ICMJE) criteria for authorship for this article, take responsibility for the integrity of the work as a whole, and have given their approval for this version to be published.

Authorship Contributions. Design of Study and Conceptualization: Lei Zha and Boris Tefsen. Data Collection: Dayan Zhang, Zhichu Ren, Xiang Li, Yi Zou, Shirong Li, Gang Yang, Shuangqi Luo and Lingling Pan. Data analysis: Lei Zha, Dayan Zhang and Lingling Pan. Original Draft Construction: Lei Zha. Draft Review and Scientific Revisions: Boris Tefsen. Final draft approval: Lei Zha, Dayan Zhang, Lingling Pan, Zhichu Ren, Xiang Li, Yi Zou, Shirong Li, Shuangqi Luo, Gang Yang, and Boris Tefsen.

Disclosures. Lei Zha, Dayan Zhang, Lingling Pan, Zhichu Ren, Xiang Li, Yi Zou, Shirong Li, Shuangqi Luo, Gang Yang, and Boris Tefsen have none to declare.

Compliance with Ethics Guidelines. The study was approved by the ethics committee of Xi'an Jiaotong-Liverpool University (reference number 19-01-05) and the institutional review board in each participating hospital, The First People's Hospital of Wuhu (approval number 202001), The Second People's Hospital of Wuhu (approval number 2019-04) and The First Affiliated Hospital of Wannan Medical College (reference number 2019-97). The patient's medical records and information were anonymized and deidentified prior to analysis.

Data availability. The datasets generated and analyzed during the current study are available from the corresponding author (Lei Zha) on reasonable request.

Open Access. This article is licensed under a Creative Commons Attribution-NonCommercial 4.0 International License, which permits any non-commercial use, sharing, adaptation, distribution and reproduction in any medium or format, as long as you give appropriate credit to the original author(s) and the source, provide a link to the Creative Commons licence, and indicate if changes were made. The images or other third party material in this article are included in the article's Creative Commons licence, unless indicated 
otherwise in a credit line to the material. If material is not included in the article's Creative Commons licence and your intended use is not permitted by statutory regulation or exceeds the permitted use, you will need to obtain permission directly from the copyright holder. To view a copy of this licence, visit http:// creativecommons.org/licenses/by-nc/4.0/.

\section{REFERENCES}

1. Brooke JS. Stenotrophomonas maltophilia: an emerging global opportunistic pathogen. Clin Microbiol Rev. 2012;25(1):2-41.

2. Looney WJ, Narita M, Mühlemann K. Stenotrophomonas maltophilia: an emerging opportunist human pathogen. Lancet Infect Dis. 2009;9(5): 312-23.

3. European Center for Disease Prevention and Control. Healthcare-associated infections in intensive care units. Annual epidemiological report for 2016. 2018.

4. Guerci P, Bellut H, Mokhtari M, Gaudefroy J, Mongardon N, Charpentier C, et al. Outcomes of Stenotrophomonas maltophilia hospital-acquired pneumonia in intensive care unit: a nationwide retrospective study. Crit Care. 2019;23(1):1-13.

5. Paez JG, Tengan F, Barone A, Levin A, Costa S, diseases i. Factors associated with mortality in patients with bloodstream infection and pneumonia due to Stenotrophomonas maltophilia. Eur J Clin Microbiol. 2008;27(10):901-6.

6. Adegoke AA, Stenström TA, Okoh AI. Stenotrophomonas maltophilia as an emerging ubiquitous pathogen: looking beyond contemporary antibiotic therapy. Front Microbiol. 2017;8:2276.

7. Chang YT, Lin CY, Chen YH, Hsueh P-R. Update on infections caused by Stenotrophomonas maltophilia with particular attention to resistance mechanisms and therapeutic options. Front Microbiol. 2015;6: 893.

8. Falagas ME, Valkimadi P-E, Huang Y-T, Matthaiou DK, Hsueh P-R. Therapeutic options for Stenotrophomonas maltophilia infections beyond co-trimoxazole: a systematic review. J Antimicrob Chemother. 2008;62(5):889-94.

9. Ko J-H, Kang C-I, Cornejo-Juárez P, Yeh K-M, Wang $\mathrm{C}-\mathrm{H}$, Cho $\mathrm{S}$, et al. Fluoroquinolones versus trimethoprim-sulfamethoxazole for the treatment of Stenotrophomonas maltophilia infections: a systematic review and meta-analysis. Clin Microbiol. 2019;25(5):546-54.

10. Matson HH, Jones BM, Wagner JL, Motes MA, Bland CM. Growing resistance in Stenotrophomonas maltophilia? Am J Health Syst Pharm. 2019;76(24): 2004-5.

11. Wu H, Wang J-T, Shiau Y-R, Wang H-Y, Lauderdale T-LY, Chang S-C, et al. A multicenter surveillance of antimicrobial resistance on Stenotrophomonas maltophilia in Taiwan. J Microbiol Immunol. 2012;45(2):120-6.

12. Farrell DJ, Sader HS, Jones RN. Antimicrobial susceptibilities of a worldwide collection of Stenotrophomonas maltophilia isolates tested against tigecycline and agents commonly used for $S$. maltophilia infections. J Antimicrob Agents Chemother. 2010;54(6):2735-7.

13. Sader HS, Farrell DJ, Flamm RK, Jones RN. Antimicrobial susceptibility of gram-negative organisms isolated from patients hospitalised with pneumonia in US and European hospitals: results from the SENTRY Antimicrobial Surveillance Program, 2009-2012. Int J Antimicrob Agents. 2014;43(4): 328-34.

14. Lu P-L, Liu Y-C, Toh H-S, Lee Y-L, Liu Y-M, Ho C-M, et al. Epidemiology and antimicrobial susceptibility profiles of gram-negative bacteria causing urinary tract infections in the Asia-Pacific region: 2009-2010 results from the Study for Monitoring Antimicrobial Resistance Trends (SMART). Int J Antimicrob Agents. 2012;40:S37-43.

15. Hu L-F, Xu X-H, Li H-R, Gao L-P, Chen $X$, Sun N, et al. Surveillance of antimicrobial susceptibility patterns among Stenotrophomonas maltophilia isolated in China during the 10-year period of 2005-2014. J Chemother. 2018;30(1):25-30.

16. Zha L, Pan L, Guo J, French N, Villanueva EV, Tefsen B. Effectiveness and safety of high dose tigecycline for the treatment of severe infections: a systematic review and meta-analysis. Adv Ther. 2020;37(3):1049-64.

17. Hawkey PM, Warren RE, Livermore DM, McNulty CA, Enoch DA, Otter JA, et al. Treatment of infections caused by multidrug-resistant gram-negative bacteria: report of the British society for antimicrobial chemotherapy/healthcare infection society/ British infection association joint working party. J Antimicrob Chemother. 2018;73(suppl_3):iii2-78.

18. Sader HS, Flamm RK, Jones RN. Tigecycline activity tested against antimicrobial resistant surveillance subsets of clinical bacteria collected worldwide 
(2011). Diagn Microbiol Infect Dis. 2013;76(2): 217-21.

19. Biagi M, Tan X, Wu T, Jurkovic M, Vialichka A, Meyer K, et al. Activity of potential alternative treatment agents for Stenotrophomonas maltophilia isolates nonsusceptible to levofloxacin and/or trimethoprim-sulfamethoxazole. J Clin Microbiol. 2020;58(2):e01603-19.

20. Tekçe YT, Erbay A, Cabadak H, Şen S. Tigecycline as a therapeutic option in Stenotrophomonas maltophilia infections. J Chemother. 2012;24(3):150-4.

21. Yuhong W, Zonghong S. High-dosage tigecycline for Stenotrophomonas maltophilia bacteremia. Chin Med J. 2014;127(17):3199.

22. Farrar JE, Garner KM, Swanson JM, Magnotti LJ, Croce MA, Wood GC, et al. Tigecycline to treat Stenotrophomonas maltophilia ventilator-associated pneumonia in a trauma intensive care unit as a result of a drug shortage: a case series. J Clin Pharm. 2020;45(4):836-9.

23. Kalil AC, Metersky ML, Klompas M, Muscedere J, Sweeney DA, Palmer LB, et al. Management of adults with hospital-acquired and ventilator-associated pneumonia: 2016 clinical practice guidelines by the Infectious Diseases Society of America and the American Thoracic Society. Clin Infect Dis. 2016;63(5):e61-111.

24. Bouadma L, Sonneville R, Garrouste-Orgeas M, Darmon M, Souweine B, Voiriot G, et al. Ventilatorassociated events: prevalence, outcome, and relationship with ventilator-associated pneumonia. Crit Care Med. 2015;43(9):1798-806.

25. Magill SS, Klompas M, Balk R, Burns SM, Deutschman CS, Diekema D, et al. Developing a new, national approach to surveillance for ventilator-associated events. Am J Crit Care. 2013;22(6):469-73.

26. CLSI. Performance standards for antimicrobial susceptibility testing; twenty-fourth informational supplement. Wayne, PA: CLSI. 2014.

27. Lodise TP, Berger A, Altincatal A, Wang R, Bhagnani $\mathrm{T}$, Gillard $\mathrm{P}$, et al. Antimicrobial resistance or delayed appropriate therapy-does one influence outcomes more than the other among patients with serious infections due to carbapenem-resistant versus carbapenem-susceptible enterobacteriaceae? Open Forum Infect Dis. 2019;6(6):ofz194.

28. De Pascale G, Montini L, Pennisi MA, Bernini V, Maviglia R, Bello G, et al. High dose tigecycline in critically ill patients with severe infections due to multidrug-resistant bacteria. Crit Care. 2014;18(3): $1-9$.
29. Cisneros JM, Rosso-Fernández CM, Roca-Oporto C, De Pascale G, Jiménez-Jorge S, Fernández-Hinojosa $\mathrm{E}$, et al. Colistin versus meropenem in the empirical treatment of ventilator-associated pneumonia (Magic Bullet study): an investigator-driven, openlabel, randomized, noninferiority controlled trial. Crit Care. 2019;23(1):1-13.

30. Frenkel WJ, Jongerius EJ, Mandjes-van Uitert MJ, van Munster BC, de Rooij SE. Validation of the Charlson Comorbidity Index in acutely hospitalized elderly adults: a prospective cohort study. J Am Geriatr Soc. 2014;62(2):342-6.

31. Niewiński G, Starczewska M, Kański A. Prognostic scoring systems for mortality in intensive care units-the APACHE model. Anaesthesiol Intensive Ther. 2014;46(1):46-9.

32. Allan V, Ramagopalan SV, Mardekian J, Jenkins A, Li X, Pan X, et al. Propensity score matching and inverse probability of treatment weighting to address confounding by indication in comparative effectiveness research of oral anticoagulants. J Comp Eff Res. 2020;9(9):603-14.

33. Austin PC, Stuart EA. Moving towards best practice when using inverse probability of treatment weighting (IPTW) using the propensity score to estimate causal treatment effects in observational studies. Stat Med. 2015;34(28):3661-79.

34. Steyerberg EW, Eijkemans MJ, Habbema JDF. Stepwise selection in small data sets: a simulation study of bias in logistic regression analysis. J Clin Epidemiol. 1999;52(10):935-42.

35. French G. A review of tigecycline. J Chemother. 2008;20(sup1):3-11.

36. Pfaller MA, Huband MD, Streit JM, Flamm RK, Sader HS. Surveillance of tigecycline activity tested against clinical isolates from a global (North America, Europe, Latin America and Asia-Pacific) collection (2016). Int $\mathrm{J}$ Antimicrob Agents. 2018;51(6):848-53.

37. Zhao J, Liu Y, Liu Y, Wang D, Ni W, Wang R, et al. Frequency and genetic determinants of tigecycline resistance in clinically isolated Stenotrophomonas maltophilia in Beijing, China. Front Microbiol. 2018;9:549.

38. Zhou H, Li G, Zhuo C, Yang Y, Shi Y, Chen B, et al. Laboratory diagnosis, clinical management and infection control of the infections caused by Stenotrophomonas maltophilia: a Chinese consensus statement. Natl Med J China. 2013;93(16):1203-13.

39. Hand E, Davis H, Kim T, Duhon B. Monotherapy with minocycline or trimethoprim/sulfamethoxazole for treatment of Stenotrophomonas maltophilia 
infections. J Antimicrob Chemother. 2016;71(4): 1071-5.

40. Papst L, Beovic B, Pulcini C, Durante-Mangoni E, Rodriguez-Bano J, Kaye KS, et al. Antibiotic treatment of infections caused by carbapenem-resistant gram-negative bacilli: an international ESCMID cross-sectional survey among infectious diseases specialists practicing in large hospitals. Clin Microbiol Infect. 2018;24(10):1070-6.

41. Giamarellou H, Poulakou G. Pharmacokinetic and pharmacodynamic evaluation of tigecycline. Expert Opin Drug Metab Toxicol. 2011;7(11):1459-70.

42. De Pascale G, Lisi L, Ciotti GMP, Vallecoccia MS, Cutuli SL, Cascarano L, et al. Pharmacokinetics of high-dose tigecycline in critically ill patients with severe infections. Ann Intensive Care. 2020;10(1): $1-9$.

43. Kontou P, Manika K, Chatzika K, Papaioannou M, Sionidou M, Pitsiou G, et al. Pharmacokinetics of moxifloxacin and high-dose levofloxacin in severe lower respiratory tract infections. Int J Antimicrob Agents. 2013;42(3):262-7.

44. Yaghoubi S, Zekiy AO, Krutova M, Gholami M, Kouhsari E, Sholeh M, et al. Tigecycline antibacterial activity, clinical effectiveness, and mechanisms and epidemiology of resistance: narrative review. Eur J Clin Microbiol Infect Dis. 2021;1-20. https:// doi.org/10.1007/s10096-020-04121-1.

45. Gutiérrez-Gutiérrez B, Salamanca E, de Cueto M, Hsueh P-R, Viale P, Paño-Pardo JR, et al. Effect of appropriate combination therapy on mortality of patients with bloodstream infections due to carbapenemase-producing Enterobacteriaceae (INCREMENT): a retrospective cohort study. Lancet Infect Dis. 2017;17(7):726-34.

46. Kohler PP, Volling C, Green K, Uleryk EM, Shah PS, McGeer A. Carbapenem resistance, initial antibiotic therapy, and mortality in Klebsiella pneumoniae bacteremia: a systematic review and meta-analysis. Infect Control Hosp Epidemiol. 2017;38(11): $1319-28$

47. Park S-Y, Park HJ, Moon SM, Park K-H, Chong YP, Kim M-N, et al. Impact of adequate empirical combination therapy on mortality from bacteremic Pseudomonas aeruginosa pneumonia. BMC Infect Dis. 2012;12(1):1-6.

48. Zilahi G, McMahon MA, Povoa P, Martin-Loeches I. Duration of antibiotic therapy in the intensive care unit. J Thorac Dis. 2016;8(12):3774-80.

49. Kollef MH, Chastre J, Clavel M, Restrepo MI, Michiels B, Kaniga K, et al. A randomized trial of 7-day doripenem versus 10-day imipenem-cilastatin for ventilator-associated pneumonia. Crit Care (London, England). 2012;16(6):R218.

50. Florescu DF, Qiu F, McCartan MA, Mindru C, Fey PD, Kalil AC. What is the efficacy and safety of colistin for the treatment of ventilator-associated pneumonia? A systematic review and meta-regression. Clin Infect Dis Off Publ Infect Dis Soc Am. 2012;54(5):670-80.

51. Kollef KE, Schramm GE, Wills AR, Reichley RM, Micek ST, Kollef MH. Predictors of 30-day mortality and hospital costs in patients with ventilator-associated pneumonia attributed to potentially antibiotic-resistant gram-negative bacteria. Chest. 2008;134(2):281-7.

52. Saied WI, Merceron S, Schwebel C, Le Monnier A, Oziel J, Garrouste-Orgeas M, et al. Ventilator-associated pneumonia due to Stenotrophomonas maltophilia: risk factors and outcome. J Infect. 2020;80(3):279-85.

53. Shah MD, Coe KE, El Boghdadly Z, Wardlow LC, Dela-Pena JC, Stevenson KB, et al. Efficacy of combination therapy versus monotherapy in the treatment of Stenotrophomonas maltophilia pneumonia. J Antimicrob Chemother. 2019;74(7):2055-9.

54. Prasad P, Sun J, Danner RL, Natanson C. Excess deaths associated with tigecycline after approval based on noninferiority trials. Clin Infect Dis. 2012;54(12):1699-709.

55. Shen F, Han Q, Xie D, Fang M, Zeng H, Deng Y. Efficacy and safety of tigecycline for the treatment of severe infectious diseases: an updated metaanalysis of RCTs. Int J Infect Dis. 2015;39:25-33.

56. Tasina E, Haidich A-B, Kokkali S, Arvanitidou M. Efficacy and safety of tigecycline for the treatment of infectious diseases: a meta-analysis. Lancet Infect Dis. 2011;11(11):834-44.

57. Bekaert M, Timsit J-F, Vansteelandt S, Depuydt P, Vésin A, Garrouste-Orgeas $M$, et al. Attributable mortality of ventilator-associated pneumonia: a reappraisal using causal analysis. Am J Respir Crit Care Med. 2011;184(10):1133-9.

58. Falagas ME, Kastoris AC, Vouloumanou EK, Rafailidis PI, Kapaskelis AM, Dimopoulos G. Attributable mortality of Stenotrophomonas maltophilia infections: a systematic review of the literature. Future Microbiol. 2009;4(9):1103-9.

59. Colpan A, Akinci E, Erbay A, Balaban N, Bodur H. Evaluation of risk factors for mortality in intensive care units: a prospective study from a referral hospital in Turkey. Am J Infect Control. 2005;33(1): $42-7$. 
60. Hanes SD, Demirkan K, Tolley E, Boucher BA, Croce MA, Wood GC, et al. Risk factors for late-onset nosocomial pneumonia caused by Stenotrophomonas maltophilia in critically ill trauma patients. Clin Infect Dis. 2002;35(3):228-35.

61. Li G, Cook DJ, Thabane L, Friedrich JO, Crozier TM, Muscedere J, et al. Risk factors for mortality in patients admitted to intensive care units with pneumonia. Respir Res. 2016;17(1):1-9.

62. Button KS, Ioannidis JP, Mokrysz C, Nosek BA, Flint J, Robinson ES, et al. Power failure: why small sample size undermines the reliability of neuroscience. Nat Rev Neurosci. 2013;14(5):365-76.

\section{Publisher's Note}

Springer Nature remains neutral with regard to jurisdictional claims in published maps and institutional affiliations. 